\title{
Exotic Vector-Like Quark Phenomenology in the Minimal Linear $\sigma$ Model
}

\section{J. Alonso-González ${ }^{a, b, *}$}

${ }^{a}$ Instituto de Física Teórica UAM/CSIC, Calle Nicolás Cabrera 13-15, Cantoblanco E-28049 Madrid, Spain

${ }^{b}$ Departamento de Física Teórica, Universidad Autónoma de Madrid, Cantoblanco E-28049 Madrid, Spain

E-mail: j.alonso.gonzalez@csic.es

Extensions of the Standard Model that include vector-like quarks commonly also include additional particles that may mediate new production or decay modes. Using as example the minimal linear $\sigma$ model, that reduces to the minimal $S O(5) / S O(4)$ composite Higgs model in a specific limit, we consider the phenomenology of vector-like quarks when a scalar singlet $\sigma$ is present. This new particle may be produced in the decays $T \rightarrow t \sigma, B \rightarrow b \sigma$, where $T$ and $B$ are vector-like quarks of charges $2 / 3$ and $-1 / 3$, respectively, with subsequent decay $\sigma \rightarrow W^{+} W^{-}, Z Z, h h$. By scanning over the allowed parameter space we find these decays may be dominant. In addition, we find that the presence of several new particles allows for single $T$ production cross sections larger than those expected in minimal models. Existing searches are sensitive to these new signatures.

40th International Conference on High Energy physics - ICHEP2020

July 28 - August 6, 2020

Prague, Czech Republic (virtual meeting)

\footnotetext{
${ }^{*}$ Speaker
} 


\section{Introduction}

The Minimal Linear $\sigma$ Model (ML $\sigma \mathrm{M})$ [1] represents the only example of a renormalisable realization embedding the Minimal Composite Higgs Model (MCHM) [2-5]. It is based on the global spontaneous symmetry breaking $S O(5) \rightarrow S O(4)$ at an energy scale $f$ and on the introduction of a real scalar $S O(5)$ quintuplet that contains as degrees of freedom the three SM GBs, the Higgs and an additional EW singlet $\sigma$. Moreover, the spectrum is enlarged with several exotic vector-like fermions in the trivial and fundamental $S O(5)$ representations that will give rise to the fermion partial compositeness mechanism [6-8] responsible of the SM fermions masses.

Based on Ref. [9], in this text it is presented the phenomenology associated with the exotic vector-like quarks (VLQs) of the ML $\sigma \mathrm{M}$. In particular, the focused is on its lightest top and bottom partners.

\section{The ML $\sigma$ M Fermionic Sector}

Following the original ML $\sigma \mathrm{M}$ proposal, that only considers third generation SM quarks and their corresponding VLQs, the renormalisable fermionic Lagrangian can be written as follows [1]

$$
\begin{aligned}
& \mathscr{L}_{f}=\overline{\mathrm{q}}_{L} i \not D \mathrm{q}_{L}+\overline{\mathrm{t}}_{R} i \not D \mathrm{t}_{R}+\overline{\mathrm{b}}_{R} i \not D \mathrm{~b}_{R} \\
& +\bar{\psi}\left[i \not D-M_{5}\right] \psi+\bar{\chi}\left[i \not D-M_{1}\right] \chi+\bar{\psi}^{\prime}\left[i \not D-M_{5}^{\prime}\right] \psi^{\prime}+\bar{\chi}^{\prime}\left[i \not D-M_{1}^{\prime}\right] \chi^{\prime} \\
& -\left[\mathrm{y}_{1} \bar{\psi}_{L} \phi \chi \chi_{R}+\mathrm{y}_{2} \bar{\psi}_{R} \phi \chi_{L}+\mathrm{y}_{1}^{\prime} \bar{\psi}_{L}^{\prime} \phi \chi_{R}^{\prime}+\mathrm{y}_{2}^{\prime} \bar{\psi}_{R}^{\prime} \phi \chi_{L}^{\prime}\right. \\
& +\Lambda_{1}\left(\overline{\mathrm{q}}_{L} \Delta_{2 \times 5} \psi_{R}\right)+\Lambda_{2} \bar{\psi}_{L}\left(\Delta_{5 \times 1} \mathrm{t}_{R}\right)+\Lambda_{3} \bar{\chi}_{L} \mathrm{t}_{R} \\
& \left.+\Lambda_{1}^{\prime}\left(\overline{\mathrm{q}}_{L} \Delta_{2 \times 5}^{\prime} \psi_{R}^{\prime}\right)+\Lambda_{2}^{\prime} \bar{\psi}_{L}^{\prime}\left(\Delta_{5 \times 1}^{\prime} \mathrm{b}_{R}\right)+\Lambda_{3}^{\prime} \bar{\chi}_{L}^{\prime} \mathrm{b}_{R}+\text { h.c. }\right]
\end{aligned}
$$

where $\mathrm{q}_{L}$ is the left-handed ( $\left.\mathrm{LH}\right) S U(2)_{L}$ doublet, $\mathrm{t}_{R}$ and $\mathrm{b}_{R}$ are the right-handed (RH) $S U(2)_{L}$ singlets, $\phi$ is the scalar quintuplet and $\psi^{(\prime)}$ and $\chi^{(\prime)}$ are the exotic VLQs in the fundamental and trivial $S O$ (5) representations, respectively. Unprimed fields are related to the top sector, whereas primed to the bottom one. The first line of Eq. (1) contains the canonical kinetic terms for the elementary quarks. The second one describes the kinetic and mass terms for the exotic quarks. The proto-Yukawa interactions among the exotic fermions and the scalar quintuplet are written in the third line. Finally, the last two lines show the $S O(5)$ breaking interactions between the exotic and the elementary quarks: the terms $\Delta_{2 \times 5}^{(\prime)}$ and $\Delta_{5 \times 1}^{(\prime)}$ denote spurion fields [10-14] that connect the exotic and the elementary sectors and are the responsible of the light fermion masses. According to the fermion partial compositeness paradigm, no direct elementary fermion couplings to $\phi$ are allowed.

In terms of the $S U(2)_{L}$ quantum numbers, the scalar quintuplet $\phi$ and the VLQs can be decomposed as follows

$$
\phi=\left(H^{T}, \widetilde{H}^{T}, \mathrm{~s}\right)^{T}, \quad \psi \sim\left(\mathrm{K}, \mathrm{Q}, \mathrm{T}_{5}\right)^{T}, \quad \chi \sim \mathrm{T}_{1}, \quad \psi^{\prime} \sim\left(\mathrm{Q}^{\prime}, \mathrm{K}^{\prime}, \mathrm{B}_{5}\right)^{T}, \quad \chi^{\prime} \sim \mathrm{B}_{1},
$$

where $H$ is the $\mathrm{SM} S U(2)_{L}$ doublet, with $\widetilde{H} \equiv i \sigma_{2} H^{*}, \mathrm{~s}$ is the scalar singlet, $\mathrm{K}^{(\prime)}$ and $\mathrm{Q}^{(\prime)}$ are $S U(2)_{L}$ doublets and $\mathrm{T}_{1,5}$ and $\mathrm{B}_{1,5}$ are singlets. The rest of the charge assignments can be seen in Table 1. 


\begin{tabular}{|c|c|c|c|c|c|c|}
\hline Charge/Field & $\mathrm{K}$ & $\mathrm{Q}$ & $\mathrm{T}_{1,5}$ & $\mathrm{Q}^{\prime}$ & $\mathrm{K}^{\prime}$ & $\mathrm{B}_{1,5}$ \\
\hline$\Sigma_{R}^{(3)}$ & $+1 / 2$ & $-1 / 2$ & 0 & $+1 / 2$ & $-1 / 2$ & 0 \\
\hline$S U(2)_{L} \times U(1)_{Y}$ & $(2,+7 / 6)$ & $(2,+1 / 6)$ & $(1,+2 / 3)$ & $(2,+1 / 6)$ & $(2,-5 / 6)$ & $(1,-1 / 3)$ \\
\hline$U(1)_{X}$ & $+2 / 3$ & $+2 / 3$ & $+2 / 3$ & $-1 / 3$ & $-1 / 3$ & $-1 / 3$ \\
\hline$U(1)_{E M}$ & $\mathrm{~K}^{u}=+5 / 3$ & $\mathrm{Q}^{u}=+2 / 3$ & $+2 / 3$ & $\mathrm{Q}^{\prime u}=+2 / 3$ & $\mathrm{~K}^{\prime u}=-1 / 3$ & $-1 / 3$ \\
& $\mathrm{~K}^{d}=+2 / 3$ & $\mathrm{Q}^{d}=-1 / 3$ & & $\mathrm{Q}^{\prime d}=-1 / 3$ & $\mathrm{~K}^{\prime d}=-4 / 3$ & \\
\hline
\end{tabular}

Table 1: Decompositions of the exotic fields and their charge assignments. The global $U(1)_{X}$ is added to ensure the correct hypercharge assignments for the SM fields. The hypercharge is given by $Y=\Sigma_{R}^{(3)}+X$, with $X$ the $U(1)_{X}$ charge and $\Sigma_{R}^{(3)}$ the third component of the global $S U(2)_{R}$, which is part of the residual $S O(4)$ group after the breaking of $S O(5)$.

Light fermion masses arise via a series of interactions $\grave{a}$ la Seesaw and once the Higgs doublets develop a VEV $v_{\mathrm{h}}=246 \mathrm{GeV}$. A first-order approximation for the values of the top and bottom quark masses is given by

$$
m_{t} \approx y_{1} \frac{\Lambda_{1} \Lambda_{3}}{M_{1} M_{5}} \frac{v_{\mathrm{h}}}{\sqrt{2}}, \quad m_{b} \approx y_{1}^{\prime} \frac{\Lambda_{1}^{\prime} \Lambda_{3}^{\prime}}{M_{1}^{\prime} M_{5}^{\prime}} \frac{v_{\mathrm{h}}}{\sqrt{2}} .
$$

\section{Constraints}

The inclusion of exotic scalar and fermionic degrees of freedom modifies the lightest states interactions with respect to the SM. These deviations can be seen in several observables that will set constraints on the parameter space. On one hand, due to the mixing between the SM bottom and its partners, the $Z \bar{b} b$-coupling deviates form the SM value and thus there are constraints from the ratios of partial widths $R_{b}$ and $R_{c}$, the forward-backward (FB) charge asymmetry $A_{\mathrm{FB}}^{b}$, and the coupling parameter $A_{b}$ from the LR FB asymmetry $[15,16]$. On the other hand, there are also bounds from $T$ and $S$ oblique parameters $[1,17,18]$, since new scalar and fermionic mass eigenstates couple to EW gauge bosons.

In addition, there are experimental and theoretical constraints to the Higgs-singlet mixing $\sin \gamma$. Bounds in the $\left(m_{\sigma}, \sin ^{2} \gamma\right)$ plane are shown in Fig. 1. Since $\sin \gamma$ universally suppresses the Higgs coupling to EW bosons, its possible to derive an upper bound from the latest ATLAS analysis of Higgs signal strengths with $80 \mathrm{fb}^{-1}$ at $\sqrt{s}=13 \mathrm{TeV}$ [19]. Using a $\chi^{2}$ fit, this bound is

$$
\left.\sin ^{2} \gamma \lesssim 0.11 \text { (at } 95 \% \text { C.L. }\right)
$$

which is highlighted by the excluded red shaded region of Fig. 1. There are also constraints from ATLAS searches for scalar resonances in diboson final states [20]. The respective 95\% C.L. limits are represented by a blue region in Fig. 1 and were obtained assuming VLQs do not contribute to the $\sigma$ gluon fusion production: only SM top quarks run in the loop with a SM-like Yukawa coupling to $\sigma$ weighted by $\sin \gamma$. It was checked this was a good approximation in most regions of the parameter space. Finally, the yellow region of Fig. 1 is theoretically forbidden in the ML $\sigma \mathrm{M}$, as there the $S O(5)$ symmetry is not spontaneously broken. 


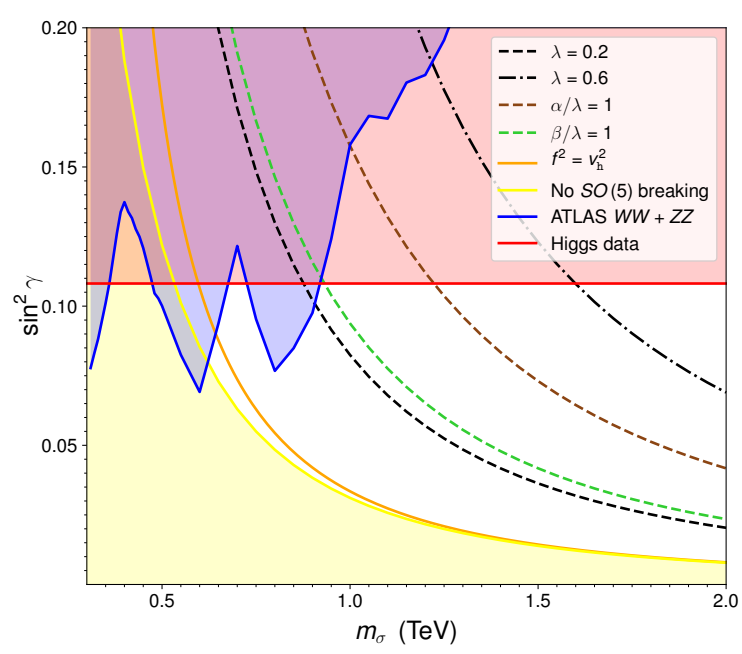

Figure 1: Constraints on the mass $\left(m_{\sigma}\right)$ and mixing angle $\left(\sin ^{2} \gamma\right)$ of the new scalar $\sigma$ (see text for details). Only the white region is allowed by both experimental and theoretical constraints. Other lines are shown where $v_{\mathrm{h}}^{2} / f^{2}=1$ and where the $S O(5)$ preserving parameter of the ML $\sigma M$ scalar potential, $\lambda$, either takes particular values or is equal to the SO(5)-breaking parameters $\alpha$ and $\beta$.

\section{Lightest VLQ Phenomenology}

The lightest VLQs of the model are phenomenologically the most interesting ones, as they would be generically the easiest to produce at colliders. Quarks with exotic charges $5 / 3$ and $-4 / 3$ are present in the model, however, since they are not coupled to the new scalar $\sigma$, its phenomenology is quite similar to that of minimal models. In the mass basis, interactions determining the production and decay of the lightest top partner $(T)$ with charge $2 / 3$ and bottom partner $(B)$ with charge $-1 / 3$ are given by

$$
\begin{aligned}
& \mathscr{L}_{G}=g_{s}\left(\bar{T} \gamma^{\mu} T+\bar{B} \gamma^{\mu} B\right) \frac{\lambda^{a}}{2} G_{\mu}^{a}, \\
& \mathscr{L}_{W Q q}=\frac{g}{\sqrt{2}}\left[\bar{T} \gamma^{\mu} V_{T b}^{L} P_{L} b+\bar{t} \gamma^{\mu} V_{t B}^{L} P_{L} B+(L \rightarrow R)\right] W_{\mu}^{+}+\text {h.c. }, \\
& \mathscr{L}_{Z Q q}=\frac{g}{2 c_{W}}\left[\bar{t} \gamma^{\mu} X_{t T}^{L} P_{L} T-\bar{b} \gamma^{\mu} X_{b B}^{L} P_{L} B+(L \rightarrow R)\right] Z_{\mu}+\text { h.c. }, \\
& \mathscr{L}_{h Q q}=\left[\bar{t} y_{t T}^{h, L} P_{L} T+\bar{b} y_{b B}^{h, L} P_{L} B+(L \rightarrow R)\right] h+\text { h.c., } \\
& \mathscr{L}_{\sigma Q q}=\left[\bar{t} y_{t T}^{\sigma, L} P_{L} T+\bar{b} y_{b B}^{\sigma, L} P_{L} B+(L \rightarrow R)\right] \sigma+\text { h.c. },
\end{aligned}
$$

where $\lambda^{a}$ are the Gell-Mann matrices, $a$ is a colour index, $G_{\mu}^{a}$ are the gluon fields, $g_{s}$ is the $S U(3)_{c}$ gauge coupling, $(L \rightarrow R)$ indicates the respective RH piece must be considered, $P_{R, L}=\left(1 \pm \gamma_{5}\right) / 2$ are the usual chirality projectors, $g$ is the $S U(2)_{L}$ gauge coupling, $c_{W} \equiv \cos \theta_{W}$, with $\theta_{W}$ the weak mixing angle, $t$ and $b$ are respectively the physical top and bottom quarks, $W$ and $Z$ are the EW gauge bosons and $h$ and $\sigma$ are the scalar mass eigenstates of the model, with $h$ the $125 \mathrm{GeV}$ Higgs. $V^{L, R}, X^{L, R}, y^{h, L, R}$ and $y^{\sigma, L, R}$ are the resulting partner couplings due to the mixing between the exotic fermions and the SM ones. Last term in Eq.(5) leads to different phenomena with respect to MCHMs and to what standard experimental analyses consider for VLQ searches: new decay modes 


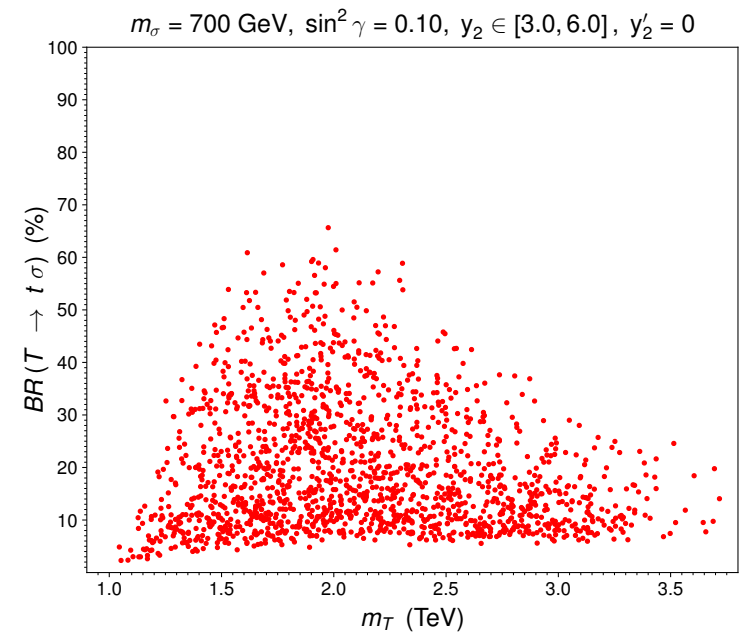

(a)

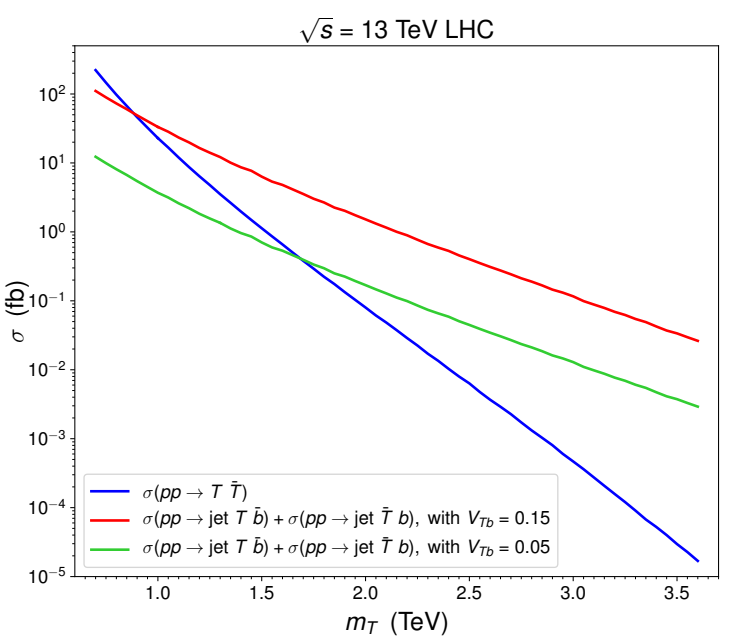

(b)

Figure 2: (a) Values of the branching ratio $B R(T \rightarrow \sigma t)$ as a function of the top partner mass $m_{T}$ for the allowed points from our MLOM parameter scan (only points where $T$ is the lightest VLQ are included). Branching ratios of $\sigma$ are approximately $B R\left(\sigma \rightarrow W^{+} W^{-}\right) \sim 40 \%, B R(\sigma \rightarrow Z Z) \sim 30 \%$, $B R(\sigma \rightarrow h h) \sim 20 \%$ and $B R(\sigma \rightarrow t \bar{t}) \sim 10 \%$. (b) $T$ pair production (blue) and single production for $V_{T b}=0.15$ (red) and $V_{T b}=0.05$ (green) cross sections at $13 \mathrm{TeV} \mathrm{LHC}$, as a function of the VLQ mass $m_{T}$.

are possible and, since $\sigma$ mainly decays to $W W, Z Z$ and $h h$, the possibility of a plethora of new multi-bosonic signatures at the LHC is opened, as long as these fermions are significantly produced through either QCD pair production or single production with the interchange of an EW boson.

As an example, Fig. 2 (a) shows the results for the $B R(T \rightarrow \sigma t)$ from a numerical scan over a region of the parameter space where $T$ is the lightest VLQ. All points are consistent with top and bottom quark masses and with bounds discussed at Sec. 3 , which are satisfied for $\mathbf{M}_{i}, \mathrm{M}_{i}^{\prime}, \Lambda_{i}$ around a few $\mathrm{TeV}$ and $\Lambda_{i}^{\prime}$ around a few hundred $\mathrm{GeV}$. As can be seen, in a significant number of scenarios with different masses the exotic decay mode is relevant and even the dominant one in few cases.

Regarding $T$ production, QCD pair production only depends on the quark mass, while the dominant contributions to single production are also controlled by $V_{T b}=\sqrt{\left(V_{T b}^{L}\right)^{2}+\left(V_{T b}^{R}\right)^{2}}$, which for some benchmarks in Fig. 2 (a) takes sizable values $V_{T b} \simeq 0.1$. However, this coupling appears to be anticorrelated with $B R(T \rightarrow \sigma t)$ : the larger $V_{T b}$, the smaller the ratio to $\sigma t$ (see Ref. [9]). Fig. 2 (b) shows $T$ pair production and single production for $V_{T b}=0.15$ and $V_{T b}=0.05$ cross sections. It becomes clear that, for $V_{T b} \simeq O(0.1)$, the single $T$ production cross section becomes more important than the pair production one above $m_{T} \simeq 1 \mathrm{TeV}$. This is a consequence of the interplay among the new VLQ states and the third generation SM quarks and represents a salient feature of realistic models as opposed to simplified VLQ models [16].

Present LHC searches are sensitive to the new decay modes. The ATLAS search for samesign di-leptons or three leptons plus $b$-tagged jets [21] can be taken as an example. This analysis primarily targets the process $p p \rightarrow B \bar{B} \rightarrow W^{-} t W^{+} \bar{t} \rightarrow W^{-} W^{+} b W^{+} W^{-} \bar{b}$, with two same-sign or three leptons produced from the decays of the $W$ bosons. The events are required to have a large amount of missing transverse energy $\boldsymbol{E}_{T}$, as well as large transverse energy $H_{T}$. This search is 
also sensitive to the processes $p p \rightarrow B \bar{B} \rightarrow \sigma b \sigma \bar{b}$ with $\sigma \rightarrow W^{+} W^{-}$, which yields the same final state as the ATLAS target. It can be shown the normalised kinematical distributions of $\boldsymbol{E}_{T}, H_{T}$ and leading lepton transverse momentum $p_{T}$ can be similar for the ATLAS target process with a standard VLQ decay mode $(B \rightarrow W t)$ and the process $p p \rightarrow B \bar{B} \rightarrow \sigma b \sigma \bar{b}$ with $\sigma \rightarrow W^{+} W^{-}$[9], and, consequently, despite being designed for a specific decay mode, the ATLAS search [21] is flexible enough to provide limits on non-standard VLQ decay modes. This and other examples (see Ref. [9]) indicate that event selections that require high $H_{T}$ or large effective mass $m_{\mathrm{eff}}=H_{T}+E_{T}$ and leptons or $b$ quarks are sensitive to the new VLQ decay channels. The results of these searches can be reinterpreted by relaxing the condition that all standard decay ratios sum to one and introducing the exotic decay modes.

\section{Acknowledgments}

The author thanks Stefano Rigolin for useful discussions. The author acknowledges partial financial support by the Spanish MINECO through the Centro de excelencia Severo Ochoa Program under grant SEV-2016-0597. The author acknowledges partial financial support by the Spanish "Agencia Estatal de Investigación" (AEI) and the EU "Fondo Europeo de Desarrollo Regional" (FEDER) through the projects FPA2016-78645-P and FPA2016-78220-C3-1-P.

\section{References}

[1] F. Feruglio, B. Gavela, K. Kanshin, P. A. N. Machado, S. Rigolin, and S. Saa, JHEP 06 (2016) 038, [arXiv: 1603.05668].

[2] K. Agashe, R. Contino, and A. Pomarol, Nucl. Phys. B719 (2005) 165-187, [hep-ph/0412089].

[3] R. Alonso, I. Brivio, B. Gavela, L. Merlo, and S. Rigolin, JHEP 12 (2014) 034, [arXiv: 1409. 1589].

[4] G. Panico and A. Wulzer, Lect. Notes Phys. 913 (2016) pp.1-316, [arXiv: 1506.01961].

[5] I. M. Hierro, L. Merlo, and S. Rigolin, JHEP 04 (2016) 016, [arXiv: 1510. 07899].

[6] M. J. Dugan, H. Georgi, and D. B. Kaplan, Nucl. Phys. B254 (1985) 299-326.

[7] D. B. Kaplan, Nucl. Phys. B365 (1991) 259-278.

[8] R. Contino and A. Pomarol, JHEP 11 (2004) 058, hep-th/0406257

[9] J. A. Aguilar-Saavedra, J. Alonso-González, L. Merlo and J. M. No, Phys. Rev. D 101, no.3, 035015 (2020) [arXiv:1911.10202 [hep-ph]].

[10] G. D’Ambrosio, G. F. Giudice, G. Isidori, and A. Strumia, Nucl. Phys. B645 (2002) 155-187, [hep-ph/0207036].

[11] V. Cirigliano, B. Grinstein, G. Isidori, and M. B. Wise, Nucl. Phys. B728 (2005) 121-134, [hep-ph/0507001].

[12] S. Davidson and F. Palorini, Phys. Lett. B642 (2006) 72-80, [hep-ph/0607329].

[13] R. Alonso, G. Isidori, L. Merlo, L. A. Munoz, and E. Nardi, JHEP 06 (2011) 037, [arXiv: 1103.5461$].$

[14] D. N. Dinh, L. Merlo, S. T. Petcov, and R. Vega-Álvarez, JHEP 07 (2017) 089, [arXiv: 1705.09284$].$

[15] P. Bamert, C. P. Burgess, J. M. Cline, D. London, and E. Nardi, Phys. Rev. D54 (1996) 4275-4300, [hep-ph/9602438].

[16] J. A. Aguilar-Saavedra, R. Benbrik, S. Heinemeyer, and M. Pérez-Victoria, Phys. Rev. D88 (2013), no. 9094010 , [arXiv: 1306.0572].

[17] C. Anastasiou, E. Furlan, and J. Santiago, Phys. Rev. D79 (2009) 075003, [arXiv: 0901 . 2117].

[18] M. Carena, E. Ponton, J. Santiago, and C. E. M. Wagner, Nucl. Phys. B759 (2006) 202-227, [hep-ph/0607106].

[19] ATLAS Collaboration, G. Aad et. al., Phys. Rev. D101 (2020) 012002, [arXiv: 1909. 02845].

[20] ATLAS Collaboration, M. Aaboud et. al., Phys. Rev. D98 (2018), no. 5 052008, [arXiv: 1808. 02380].

[21] ATLAS Collaboration, M. Aaboud et. al., JHEP 12 (2018) 039, [arXiv: 1807. 11883]. 